\title{
TAGUNG
}

\section{Der Einfluss von Politikern und Institutionen auf die Finanzpolitik von Staaten, Regionen und Kommunen}

\author{
Frédéric Krumbein und Julian Plottka*
}

Friedrich Heinemann eröffnete die Konferenz und stellte fest, dass zwei Säulen die Analyse der fiskalischen Politik in Europa kennzeichnen. Zum einen analysiere die Wirtschaftswissenschaft die Politik zur Stabilisierung der politischen und wirtschaftlichen Institutionen in der Europäischen Union. Zum anderen werde die Rolle der Politikerinnen und Politiker bei der Bewältigung der Finanz- und Schuldenkrise untersucht.

Überraschende Erkenntnisse aus der USPolitik

Agustin Casas untersuchte, wie politische Kandidaten durch Vorwahlen in den USA ausgesucht werden. Zwei typische Auswahlkriterien seien deren ideologische Ausrichtung und ihre Wählbarkeit, zum Beispiel ihr Charisma oder ihre Fähigkeit Spenden zu sammeln. Die zentrale - auf den ersten Blick kontraintuitive - These von Casas war, dass offene Vorwahlen, in denen jeder Bürger abstimmen kann, zu ideologisch extremeren Kandidaten führen.

In geschlossenen Vorwahlen, in denen nur Parteianhänger wählen dürfen, würden hingegen Kandidaten ausgesucht, die sich stärker in der politischen Mitte verorten. Dies liege daran, dass parteigebundene Wähler stärker wünschten, dass ihr Kandidat gewinne. Aus diesem Grund seien sie eher bereit, die Wählbarkeit des Kandidaten vor ihre politische Präferenz zu stellen. In offenen Vorwahlen seien die Wähler weniger engagiert und ständen der möglichen

\section{Fiscal Performance: The Role of Institutions And Politicians}

\section{1.-12. April 2013, Mannheim}

Gemeinsame Tagung des Arbeitskreises Europäische Integration e.V. (AEI), des Sonderforschungsbereichs „Politische Ökonomie von Reformen“ an der Universität Mannheim und des Zentrums für Europäische Wirtschaftsforschung (ZEW), Mannheim

Mit freundlicher Unterstützung der Europäischen Kommission

Elections and Partisan Politics

Chair: Friedrich HEINEMANN, ZEW Mannheim

Partisan politics: parties, primaries and elections Agustin CASAS, Carlos III University of Madrid

Discussion: Andreas BERNECKER, University of Mannheim

Divided We Reform? Evidence from US Welfare Policies

Andreas BERNECKER, University of Mannheim

Discussion: Agustin CASAS, Carlos III University of Madrid

Keynote Lecture: Fiscal Policy, Financial

Markets, and Institutions

Thomas STRATMANN, George Mason University, Fairfax

Subnational Fiscal Rules

Chair: Mustafa YETER, ZEW Mannheim

* Dr. Frédéric Krumbein, Geschäftsführer des Arbeitskreises Europäische Integration, Berlin. Julian Plottka, Wissenschaftlicher Mitarbeiter am Institut für Europäische Politik, Berlin. 
Wählbarkeit von Kandidaten gleichgültiger gegenüber.

Wenn ein Kandidat wenig Charisma besitze, aber trotzdem in den Vorwahlen gewinne, sei es wahrscheinlich, dass er sich sehr nah an der politischen Präferenz des ,median voters' befinde. Wenn ein Kandidat hingegen über viel Charisma verfüge und ausgewählt werde, sei es wahrscheinlich, dass er weiter von der Position des ,median voter' entfernt sei.

Andreas Bernecker beantwortete in seinem Vortrag die Frage, ob ,divided governments', also Regierungen, die eine andere politische Zugehörigkeit als die Mehrheit im Parlament haben, weniger Sozialreformen durchführen als Regierungen, die sich auf eine eigene Mehrheit stützen können. Die Analyse konzentrierte sich auf die US-Bundesstaaten im Zeitraum von 1978 bis 2010. Bernecker fand heraus, dass , divided governments' mit einer um 25 bis 50 Prozent höheren Wahrscheinlichkeit Sozialreformen durchführen.

\section{Erklärungen und Lösungen für die Schuldenkrise in Europa}

Thomas Stratmann sprach in seinem Vortrag über die Zinssätze von Staatsanleihen und die Gründe für deren Unterschiede bei europäischen Staatsanleihen. Beispielsweise bezahle Griechenland aktuell etwa zehnmal so hohe Zinsen wie Deutschland.

Gründe für das unterschiedliche Zinsniveau lägen im fiskalischen Bereich in erster Linie in den von den Ländern umgesetzten Austeritätsmaßnahmen, das sind Sparmaßnahmen und Steuererhöhungen. Kürzungen im Sozialbereich, bei staatlichen Transferleistungen und Löhnen im öffentlichen Dienst führten eher zu niedrigeren Zinsraten als Steuererhöhungen oder Kürzungen öffentlicher Investitionen. Kürzungen für die es schwieriger ist, eine Mehrheit zu gewinnen, wie Sparmaßnahmen im Sozialstaat, signalisierten den Finanzmärkten eine starke Bereitschaft der Regierung, ihren Haushalt zu konsolidieren.
Sub-national deficits in European countries: The impact of fiscal rules and tax autonomy

Dirk FOREMNY, University of Barcelona

Discussion: Saleem A. BAHAJ, University of Cambridge

The causes and effects of US State fiscal rules: evidence from a panel study

Saleem A. BAHAJ, University of Cambridge

Discussion: Mustafa YETER, ZEW Mannheim

Sovereign Bond Market Reactions to Fiscal Rules and No-Bailout Clauses - The Swiss Experience

Marc-Daniel MOESSINGER, ZEW Mannheim

Discussion: Dirk FOREMNY, University of Barcelona

\section{Selection of Politicians}

Chair: Florian MISCH, ZEW Mannheim

Moonlighting Politicians: Motivation Matters!

Alessandro FEDELE, Free University of Bolzano

Discussion: Pierpaolo GIANNOCCOLO, University of Bologna

Political Selection and the Concentration of Political Power

Emanuel HANSEN, University of Cologne

Discussion: Alessandro FEDELE, Free University of Bolzano

Moneycracy

Pierpaolo GIANNOCCOLO, University of Bologna

Discussion: Emanuel HANSEN, University of Cologne

\section{National Fiscal Rules}

Chair: Sarah CIAGLIA, Federal Ministry of Finance, Berlin

The New EU Fiscal Governance Framework: A Quantum Leap or only Small Steps ahead

Gerrit B. KOESTERS, European Central Bank, Frankfurt

Discussion: Sarah CIAGLIA, Federal Ministry of Finance, Berlin

The Design of National Fiscal Frameworks and their Budgetary Impact

Wolf Heinrich REUTER, Vienna University of Economics and Business, Vienna 
Weitere institutionelle Gründe für unterschiedliche Zinsraten seien unter anderem das Niveau der Korruption in einem Staat. Das Niveau der Zinsraten werde außerdem durch die Garantie bürgerlicher Rechte durch den Schuldnerstaat positiv beeinflusst, weniger durch politische Rechte, wie dem Wahlrecht.

In der Europäischen Union seien die Zinsraten vor Einführung des Euro unterschiedlich hoch gewesen. Nach seiner Einführung hätten sich diese stark angenähert, weil die Märkte die falsche Illusion gehegt hätten, dass die Risiken in allen Eurostaaten ähnlich sind. Nach der Krise hätten sich die Zinsraten wieder den unterschiedlichen finanziellen und wirtschaftlichen Gegebenheiten der verschiedenen Eurostaaten angepasst.

Gründe für die hohen Zinsraten in Griechenland fänden sich in einer schlechten Fiskalpolitik, weitverbreiteter Korruption und zu viel Bürokratie. Italien und Spanien zeichneten sich durch eine niedrige Wettbewerbsfähigkeit aus.

Die Lösung der europäischen Schuldenkrise könnte in einer umfassenden Garantie Deutschlands für die Schulden der anderen Eurostaaten liegen, zum Beispiel durch die Einführung von Eurobonds. Ebenfalls denkbar wäre eine höhere Inflation, die aber ebenfalls einer Umverteilung gleichkäme. Eine Fortführung der gegenwärtigen Austeritätspolitik oder die Aufgabe des Euro durch die nicht wettbewerbsfähigen Staaten seien weitere mögliche Alternativen.

\section{Die Wirkung fiskalischer Regeln auf der subnationalen Ebene}

In der folgenden ,paper session" wurde die auf EU-Ebene diskutierte Frage, wie sich die Verschuldung von Staaten begrenzen lässt, auf die subnationale Ebene übertragen. Die vorgestellten Papiere untersuchten an den Beispielen der Mitgliedstaaten der ,EU-15', der Schweiz und der USA die Wirkung unterschiedlicher Typen fiskalischer Vorschriften auf die Verschuldung der subnationalen Ebenen.
Discussion: Peter CLAEYS, University of Barcelona

On the identification of fiscal policy rules Peter CLAEYS, University of Barcelona

Discussion: Wolf Heinrich REUTER, Vienna University of Economics and Business, Vienna

Personal Characteristics of Politicians

Chair: Marc-Daniel MOESSINGER, ZEW Mannheim

Do Lawyer-Legislators Protect Their Business? Evidence from Voting Behavior on Tort Reforms Ulrich MATTER, University of Basel

Discussion: Florian NEUMEIER, Philipps University Marburg

Political Leaders' Socioeconomic Background and Public Budget Deficits: Evidence from OECD Countries

Florian NEUMEIER, Philipps University Marburg

Discussion: Sebastian THOMASIUS, Free University of Berlin

Voters prefer more qualified mayors, but does it matter for public finances?

Sebastian THOMASIUS, Free University of Berlin

Discussion: Ulrich MATTER, University of Basel

Formation of Policy Decisions

Chair: Eckhard JANEBA, University of Mannheim

Composition Effects of the German Federal Government on the Average Top Income Tax Burden Katrin SCHARFENKAMP, University of Münster

Discussion: Christoph SCHRÖDER, ZEW Mannheim

What lies behind the promise of fiscal austerity? Unveiling the determinants of party positioning in EU countries

Alessandro SAPIO, Parthenope University of Naples

Discussion: Katrin SCHARFENKAMP, University of Münster

Will the German Debt Brake Succeed? Survey Evidence from State Politicians

Christoph SCHRÖDER, ZEW Mannheim

Discussion: Alessandro SAPIO, Parthenope University of Naples 
Dirk Foremny differenziert zwischen expliziten und impliziten fiskalischen Regeln. Erstere versuchten, eine Verschuldung durch rechtliche Vorschriften zu begrenzen. Letztere meinten die Gewährung finanzieller Autonomie und Eigenverantwortung für Schulden. Ihre Wirkung sei in Zentral- und Föderalstaaten unterschiedlich. Während in Zentralstaaten explizite Regeln zu einer geringeren Verschuldung der subnationalen Ebene führten, hätte in Föderalstaaten finanzielle Autonomie eine bessere Wirkung. In seiner Analyse der ,EU-15'Mitgliedstaaten (1995 bis 2008) konkretisierte er, dass das institutionelle Design fiskalischer Regeln, der Budgetinstitutionen und der Steuersysteme Einfluss auf die Verschuldung habe. Auch die unterschiedliche Regeldurchsetzung auf kommunaler Ebene spiele eine Rolle, lasse sich anhand der verfügbaren Daten jedoch nicht untersuchen.

Auch Marc-Daniel Moessinger unterschied in seiner Untersuchung kommunaler Finanzen in 18 schweizer Kantonen (1981 bis 2001) zwischen (expliziten) fiskalischen Regeln und einer glaubwürdigen ,no-bailout'-Regel zur Begrenzung von Schulden. Er fragte, welches Instrument besser geeignet ist, um das Vertrauen der Märkte in nachhaltige öffentliche Finanzen zu schaffen. Indikator waren die Refinanzierungskosten: Diese seien umso höher, je geringer das Vertrauen ist. Schweizer Kommunen böten sich als Untersuchungsfall an, da seit der Zahlungsunfähigkeit der Gemeinde Leukerbad Ende der 1990er Jahre und einem Gerichtsurteil eine glaubwürdige ,no-bailout'Regel besteht. In den Jahren davor sei erwartet worden, dass Kantone für überschuldete Kommunen einstehen. Die Analyse kommt zu dem Ergebnis, dass sowohl fiskalische als auch die ,no-bailout'-Regel einen positiven Effekt auf das Vertrauen der Märkte und damit auf die Refinanzierungskosten haben.

Saleem A. Bahaj differenzierte weitergehend zwischen vier Typen fiskalischer Regeln: Exante-Regeln, die während der Budgetaufstellung Anwendung finden; Kontrollen innerhalb des laufenden Haushaltsjahrs; Ex-post-Regeln,

\section{Finance Ministers and Institutional Settings}

Chair: Marc-Daniel MOESSINGER, ZEW Mannheim

The Effect of the Finance Minister's Ideology and Fiscal Rules on Tax Revenue Projections: Evidence from Swiss cantons

Florian CHATAGNY, Swiss Federal Institute of Technology Zurich

Discussion: Ronny FREIER, German Institute for Economic Research (DIW), Berlin

Dynamic Linkage between Politician Attributes and Debt Sustainability in Africa

Uchenna EFOBI, Covenant University, Nigeria

Discussion: Marc-Daniel MOESSINGER, ZEW Mannheim

When Can We Trust Population Thresholds in Regression Discontinuity Designs?

Ronny FREIER, German Institute for Economic Research (DIW), Berlin

Discussion: Florian CHATAGNY, Swiss Federal Institute of Technology Zurich

Keynote Lecture: Men Vote in Mars, Women Vote in Venus: A Survey Experiment in the Field Vincenzo GALASSO, University of Italian Switzerland, Lugano

\section{Fiscal Institutions}

Chair: Lars P. FELD, University of Freiburg

Vertical grants and local public efficiency

Peter HAUG, Halle Institute for Economic Research (IWH), Halle

Discussion: Florian MISCH, ZEW Mannheim

Fiscal adjustments and the probability of sovereign default

Martin WEDER, University of Lucerne

Discussion: Christoph ESSLINGER, University of Mannheim

die unter anderem die weitere Kreditaufnahme begrenzen; sowie , budget stabilisation funds'. In diesen werden in Zeiten hoher Einahmen Gelder angespart, um sie in Krisenzeiten einsetzen zu können, ohne Schulden aufzuneh- 
men. Die für den Zeitraum 1978 bis 2007 untersuchten US-Bundesstaaten verfügten über große finanzielle Autonomie und erlegten sich fiskalische Regeln selbst auf. Die Untersuchung zeigte, dass in normalen Zeiten Ex-postRegeln zu den besten Ergebnissen führen, während bei Steuerschocks ,budget stabilisation funds` die stärkste Wirkung haben.

\section{Die schwierige Messung der Qualität von Politikern}

Alessandro Fedele unterschied zwei Typen von Politikern: Politiker, die nur in der Politik erfolgreich sind (politisch orientierte Politiker) und solche, die auch in der Privatwirtschaft erfolgreich sind (privatwirtschaftlich orientierte Politiker). Letztere arbeiteten neben ihrer politischen Tätigkeit möglichst weiter, um ein höheres Gesamteinkommen zu erzielen.

Die Studie habe die Mitglieder der beiden Kammern des italienischen Parlaments für die Jahre 1996 bis 2006 untersucht. Dabei seien nur die neugewählten Mitglieder und diejenigen, die ohne vorherige politische Erfahrung gewählt wurden, untersucht worden. Es habe sich herausgestellt, dass die privatwirtschaftlich orientierten Politiker höhere Abwesenheitsraten im Parlament aufweisen. Außerdem hätten sie höhere Einkommen als die politisch orientierten Politiker. Die privatwirtschaftlich orientierten Politiker gingen oftmals in die Politik, um ihre privatwirtschaftlichen Karrierechancen zu verbessern beziehungsweise ihr eigenes Unternehmen zu fördern. Dabei bestehe häufig die Gefahr von Interessenskonflikten. Wenn Nebentätigkeiten von Politikern verboten würden, bestünde aber die Gefahr, dass in der Privatwirtschaft erfolgreiche Bürger der Politik ganz fern blieben.

Emanuel Hansen befasste sich mit der Selektion von Politikern und der Konzentration politischer Macht. Er stellte sich die Frage, ob eine Konzentration politischer Macht ökonomisch sinnvoll sei oder nicht. Seit Langem sei bekannt, dass eine hohe Verteilung von Macht auf viele verschiedene Akteure positiven Einfluss auf die wirtschaftliche Entwicklung ausübe. Eine hohe Qualität der Politiker, das heißt eine starke Fokussierung auf politische Ziele und deren Umsetzung, lasse hingegen eine Konzentration politischer Macht wünschenswert erscheinen. Politische Macht sollte vor allem dann begrenzt und breit verteilt werden, wenn die Motivation von Politikern für ihre politische Tätigkeit in erster Linie aus ihren Ämtern und den damit verbundenen Machtbefugnissen und Privilegien resultiere und nicht aus dem Wunsch politischer Gestaltung.

Pierpaolo Giannoccolo referierte über Möglichkeiten, die Qualität von Politikern zu analysieren und konzentrierte sich dabei auf die Gehälter von Politikern in den Staaten der OECD. ${ }^{1}$ Die Studie zeige, dass die besten Politiker nicht die höchsten Gehälter verlangten, sondern andere Motivationen hätten. Höhere Gehälter für Politiker garantierten nicht die besten Politiker. Italien sei ein Beispiel für dieses Phänomen: Abgeordnete verdienten in Italien im Vergleich der OECD-Länder am meisten, aber verfügten gleichzeitig über niedrigere Bildungsabschlüsse als in anderen OECDStaaten. Es befänden sich auch weniger Frauen in den beiden Kammern des italienischen Parlaments als in denen anderer Staaten.

\section{Einflussfaktoren auf die Haushaltspolitik der nationalen Ebene}

Mit dem Europäischen Semester, dem Fiskalvertrag, dem Euro-Plus-Pakt, dem ,two pack sowie dem , six pack' ist ein vielfältiges Instrumentarium zur Kontrolle nationaler Politik in der Eurozone geschaffen worden, das in der öffentlichen Debatte als zu strikt kritisiert wird. Gerrit B. Koesters erwiderte diese Kritik auf Basis einer Analyse der Wirkung des in den 1990er Jahren geschaffenen Stabilitäts- und Wachstumspakts (SWP) sowie der seitdem neu hinzugekommenen Instrumente. Der SWP sei von den Mitgliedstaaten nicht eingehalten wor-

1 Organisation for Economic Co-operation and Development. 
den und habe nicht funktioniert. Die neuen Instrumente würden den SWP stärken, jedoch sei der Fortschritt begrenzt, da die Kriterien sehr weich seien und es viele Schlupflöcher gebe. Sowohl automatische Verfahren als auch glaubhafte Sanktionen fehlten bis heute, sodass die Umsetzung weiterhin vom politischen Willen abhängt.

Wolf Heinrich Reuter untersuchte die Wirkung numerischer fiskalischer Regeln sowie nationaler Finanzräte auf die Nachhaltigkeit öffentlicher Finanzen auf der Einnahme- und Ausgabenseite. Finanzräte sind unabhängige Beratungsorgane, die Finanzpolitik im Sinne der Nachhaltigkeit beeinflussen. Bei den numerischen fiskalischen Regeln unterschied Reuter zwischen Defizit-, Einnahme- und Ausgaberegeln sowie Vorgaben für einen ausgeglichenen Haushalt. Während die beiden letzten Regeltypen am häufigsten vorkommen, wurden die Einnahmeregeln aufgrund einer zu kleinen Fallzahl nicht in die Analyse einbezogen. Diese zeigte, dass das Vorhandensein einer Regel einen positiven Effekt auf der Einnahme- wie Ausgabenseite hat, der sich verstärke, wenn die Regel Verfassungsrang besitzt. Finanzräte hingegen hätten nur auf der Ausgabenseite Wirkung. Den stärksten Effekt zeigten Kombinationen aus Fiskalregel und Finanzrat.

Während sich die beiden anderen Papiere mit der Wirkung fiskalischer Institutionen auf die Politik befassten, untersuchte Peter Clayes den Zusammenhang zwischen öffentlichen Finanzen und der konjunkturellen Entwicklung. In der Theorie sollten Regierungen als externe Akteure in ihrem Handeln nicht dem Konjunkturzyklus folgen. In der Praxis würden Regierungen in Zeiten des Booms aber nicht versuchen, die wirtschaftliche Entwicklung zu dämpfen. Als Ursachen für dieses Verhalten von Regierungen identifizierte er politischen Wettbewerb, die Verzögerung durch die Dauer des Haushaltsprozesses sowie im Falle von Entwicklungsstaaten den besseren Zugang zu Krediten in Boomphasen.
Der Einfluss der Eigenschaften von Politikern auf ihre Politik

Ulrich Matter analysierte, ob Parlamentarier in den USA, die Anwälte sind, dazu neigten, ihre eigenen geschäftlichen Interessen in der Gesetzgebung zu schützen. Eine wichtige Einnahmequelle von Rechtsanwälten in den USA stellten Entschädigungen von Unternehmen an Konsumenten dar, die durch Produkte geschädigt wurden (,tort laws'). Zahlungen von Tabakkonzernen an Bürger seien ein solches Beispiel. Seit den 1980er Jahren habe es in den USA einige Reformen gegeben, welche die Klagemöglichkeiten der Konsumenten eingeschränkt hätten.

Zwischen 1995 und 2012 habe es 54 Abstimmungen im Kongress sowie in den Bundesstaaten gegeben, die Einschränkungen von Klagemöglichkeiten der Konsumenten beziehungsweise Begrenzungen der Höhe von Entschädigungen zum Inhalt gehabt hätten. Matter stellte fest, dass Parlamentarier, die Rechtsanwälte sind, signifikant häufiger gegen solche Einschränkungen als Nichtanwälte gestimmt hätten. Damit tendierten sie dazu, ihre eigenen geschäftlichen Interessen zu schützen.

Florian Neumeier diskutierte die Frage, welche Rolle der sozioökonomische Status von Regierungschefs für ihre Finanz- und Wirtschaftspolitik spielt. Menschen mit niedrigem sozialem Status antizipierten nach dem Stand der Forschung weniger die zukünftigen Folgen ihrer gegenwärtigen Entscheidungen. Neumeiers Hypothese besagte, dass politische Führer mit einem ursprünglich niedrigen sozioökonomischen Status stärker eine schuldenfinanzierte Politik betrieben. Die Hypothese habe er mit Daten aus 21 OECD-Ländern (1980 bis 2008) getestet. Der sozioökonomische Status der Regierungschefs sei durch den „International Socio-Economic Index of Occupational Status" gemessen worden, der jedem Beruf einen bestimmten Zahlenwert zuordne, abhängig von Einkommen und Reputation.

Insgesamt gebe es einen Zusammenhang zwischen dem sozioökonomischen Status der Re- 
gierungschefs und der Schuldenquote: Regierungschefs mit niedrigem sozioökonomischem Status erhöhten die Schulden stärker als Regierungschefs mit hohem Status.

Sebastian Thomasius referierte über den Einfluss des Berufs der Bürgermeister der 2.031 bayrischen Kommunen auf die Wahlergebnisse und Finanzpolitik. Auf den Wahlzetteln in Bayern sei der Beruf hinter dem Namen vermerkt.

Studien aus den USA und Spanien zeigten, dass die Nennung des Berufs auf den Wahlzetteln einen Einfluss auf die Wahlchancen der Kandidaten hat. Thomasius untersuchte, ob Kandidaten mit Berufen, die einen Universitätsabschluss voraussetzen, höhere Wahlchancen haben. Bei Wahlen erhielten diese tatsächlich im Schnitt 2,6 Prozentpunkte mehr. Amtsinhaber verfügten ebenfalls über einen Bonus.

In größeren Kommunen verstärkten sich diese beiden Effekte, vermutlich weil die Wähler die Kandidaten nicht persönlich kennen. Ein weibliches Geschlecht verringere die Wahlchancen deutlich. Eine Frau erhalte im Schnitt fünf Prozentpunkte weniger als ein Mann.

Bei der Politik der Bürgermeister gebe es einen signifikanten Zusammenhang zwischen deren Berufsgruppe und ihrer Fiskalpolitik: Bauern erhöhten stärker lokale Steuern als andere Berufsgruppen und seien insgesamt fiskalkonservativer. Ein weiteres Ergebnis der Studie sei, dass Amtsinhaber stärker die Schulden reduzierten als neugewählte Bürgermeister.

\section{Politikermerkmale und Schuldenstand}

Florian Chatagny untersuchte, wie die parteipolitische Zugehörigkeit des Finanzministers die Steuerpolitik in den schweizer Kantonen beeinflusst. Dabei hat er sich auf die Haushaltsschätzung der Finanzminister konzentriert, das heißt wie diese die Haushaltseinnahmen für das nächste Jahr kalkulieren. Eine Überschätzung der Einnahmen würde zu höheren Ausgaben führen, die dann nicht gedeckt wären. Die Schätzungen des künftigen Haushalts variierten stark von Kanton zu Kanton. Nur der Kanton Bern hätte seinen Haushalt unterschätzt. Alle anderen hätten ihre Einnahmen deutlich überschätzt, teilweise bis zu 200 Franken per capita. Ein Ergebnis der Studie besage: Je konservativer ein Finanzminister sei, desto weniger stellten sich die Haushaltsschätzungen übertrieben optimistisch dar.

Uchenna Efobi sprach über den Zusammenhang zwischen Attributen von Politikern und der Staatsverschuldung in Afrika. Die meisten existierenden Studien konzentrierten sich auf die Attribute der Finanzminister. Die Studie von Efobi beschäftigte sich mit den Eigenschaften der Staats- und Regierungschefs, weil diese die Richtlinien der Politik festlegen und die Finanzminister berufen und entlassen. Die Studie untersuchte 39 afrikanische Staaten im Zeitraum von 1996 bis 2010.

Die abhängige Variable sei das Verhältnis der multilateralen Staatsverschuldung, zum Beispiel von Schulden bei der Weltbank, zur gesamten Staatsverschuldung. Die unabhängige Variable bestünde in den Attributen von Politikern, soziodemografischen Faktoren, dem Bildungsniveau, der politischen Erfahrung und ihrer parteipolitischen Zugehörigkeit. Bei den soziodemografischen Faktoren hat Efobi das Geschlecht, die Zahl der Kinder, das Alter und den Familienstand analysiert. Beim Bildungsniveau sei untersucht worden, ob die Staatsund Regierungschefs eine wirtschaftswissenschaftliche Ausbildung besitzen.

Die zentralen Ergebnisse seien, dass ältere Staatschefs weniger Schulden akkumulierten ebenso wie Staatschefs mit Kindern. Dies erkläre sich aus der afrikanischen Tradition, dass Eltern ihren Kindern keine Schulden hinterließen. Politiker, die zu keiner Partei gehörten, würden ebenfalls weniger Schulden aufnehmen. Außerdem würden politische Erfahrung und eine lange Amtsdauer zu einer geringeren Schuldenaufnahme führen.

Insgesamt lägen die Gründe für Staatsverschuldung in Afrika in einem Versagen der In- 
stitutionen, in Korruption und Missmanagement sowie in mangelhaften Fähigkeiten der politischen Führer.

Ronny Freier sprach über die Frage, ob eine höhere Anzahl von Parlamentariern zu höheren Staatsausgaben führt. Dabei untersuchte er, inwieweit Veränderungen der Einwohnerzahl, die die das Parlament vergrößerten, Auswirkungen auf die Staatsausgaben haben. So gebe es in vielen Staaten und auf unterschiedlichen politischen Ebenen eine Kopplung der Zahl der Parlamentarier an die Einwohnerzahl. Die Studie von Freier untersuchte die Bevölkerungsgrenzen in bayrischen Kommunen. Bei einer Überschreitung bestimmter Schwellenwerte bei der Bevölkerungszahl steige automatisch die Zahl der Gemeinderatsmitglieder.

Die Größe der Gemeinderäte variiere zwischen acht und 60 Mitgliedern. Weitere Änderungen in den Kommunen, wie die Bezahlung des Bürgermeisters oder sein Status als Teil- oder Vollzeitangestellter, hingen ebenfalls an den Einwohnerzahlen. Die Kommunen erhöben selbst ihre Bevölkerungszahlen. Die Datenbasis bildeten 2.056 Kommunen in Bayern im Zeitraum 1983 bis 2004. Es gebe insgesamt keinen signifikanten Zusammenhang zwischen der Zahl der Gemeinderatsmitglieder und der Höhe der Ausgaben in den Kommunen.

\section{Der Einfluss politischer Orientierung und biografischer Merkmale von Politikern auf die öffentlichen Finanzen}

Katrin Scharfenkamp untersuchte, ob ein $\mathrm{Zu}-$ sammenhang zwischen biografischen Merkmalen der Mitglieder deutscher Bundesregierungen und der Höhe des Spitzensteuersatzes besteht. Auf Basis der Annahme eigennützig handelnder Politiker war die Ausgangshypothese des vorgestellten Papiers, dass der Spitzensteuersatz niedriger sei, je höher die gesellschaftliche ,Klasse` der Regierungsmitglieder ist. Dieser Zusammenhang sei für die USA nachweisbar. Untersuchungsgegenstand waren die Mitglieder der Bundesregierungen und der Spitzensteuersatz seit 1957. ${ }^{2}$ Als unabhängige Variablen wurden die gesellschaftliche ,Klasse“ (Beruf des Vaters), der höchste Bildungsabschluss, die Erfahrung in Führungspositionen außerhalb der Politik und das Alter der Regierungsmitglieder untersucht. Scharfenkamp musste jedoch feststellen, dass auf dieser Datenbasis die Hypothese weder bestätigt noch widerlegt werden kann und weitere Forschung notwendig sei.

Auch Alessandro Sapio ging in seiner Untersuchung über die institutionellen Rahmenbedingungen als Einflussfaktoren auf die öffentlichen Finanzen hinaus und untersuchte die Relevanz der politischen Positionen der Parteien auf wirtschaftliche Orthodoxie in der Regierungspolitik. Diese definierte er als Senkung der nationalen Schulden, eine starke Währung und die Unterstützung des Bankensystems. Die politischen Positionen der Parteien in den Mitgliedstaaten der ,EU-15' (1990 bis 2006) erhob er mithilfe des Datensatzes des „Manifesto Research Group/Comparative Manifestos Project (MRG/CMP)“. Als Ergebnis seiner Untersuchung konnte er festhalten, dass linke und rechte Parteien in ihrer Politik unterschiedlich auf makroökonomische Schocks reagieren. Parteiübergreifend habe jedoch die Dauer der Amtszeit einen negativen Einfluss auf Sparpolitik, genauso habe die Fragmentierung des Parteiensystems Einfluss, da durch sie der politische Wettbewerb steige.

Christoph Schröder wandte sich in dem von ihm präsentierten Papier dem institutionellen Rahmen deutscher Haushaltspolitik zu, der ab 2016 im Bund und ab 2020 in den Bundesländern geltenden Schuldenbremse. ${ }^{3}$ Auf Basis eine Umfrage unter 1.861 Mitgliedern der Parlamente der Bundesländer zu deren Erwartung, ob die Schuldenbremse eingehalten werden wird, sei untersucht worden, unter welchen Be-

2 Die Einkommenssteuer wird auf Bundesebene seit 1958 erhoben, für das Entscheidungsverfahren zur Festlegung der Steuersätze hat die Referentin ein Jahr veranschlagt.

3 Art. 105 und 119 Grundgesetz. 
dingungen die Bundesländer die Vorgaben einhalten werden. Während ein Einfluss der parteipolitischen Zugehörigkeit der Antwortenden nicht nachweisbar sei, zeige sich ein ,overconfidence bias' unter den Mitgliedern der jeweiligen Regierungsparteien und in bestimmten Bundesländern, dass das jeweilige Bundesland beziehungsweise Regierung die Schuldenbremse einhalten werde.

\section{Hat der Erfolg negativer Wahlkampagnen einen, gender bias'?}

In seiner ,keynote speech' widmete sich Vincenzo Galasso der Wirkung negativer Wahlkampagnen auf das Wahlverhalten und die Wahlbeteiligung. Dazu präsentierte er die Ergebnisse eines Forschungsprojekts, das 2011 parallel zu den Kommunalwahlen in Mailand stattfand. Ausgehend von den Thesen neuropsychologischer Forschung, dass Frauen und Männer unterschiedlich auf positive und negative Anreize reagierten, sollte überprüft werden, ob Wählerinnen und Wähler unterschiedlich auf kompetitive Wahlkämpfe reagieren.

Dazu wurden zwei Wochen vor der Wahl mehr als 400 Probanden in zwei Wellen mit gezielter Wahlwerbung (erste Welle: Kandidateninterview und Wahlslogan; zweite Welle: offener Brief an die Wähler und Kandidatenvideo) bespielt. Eine Gruppe wurde positiver, eine Gruppe negativer und eine Kontrollgruppe keiner gezielten Wahlwerbung ausgesetzt. In einer dritten Welle wurden die Probanden nach der Wahl über ihr Wahlverhalten und ihre Erwartung hinsichtlich des Ergebnisses der folgenden Stichwahl befragt.

Für die Gesamtgruppe sei kein Einfluss des Experiments auf das Wahlverhalten festzustellen. Jedoch hat sich unter den befragten Frauen das positive , campaigning' für den Gegenkandidaten des Amtsinhabers positiv ausgewirkt. Das negative ,campaigning' hat unter den befragten Frauen die Wahlbeteiligung gesenkt und unter den befragten Männern erhöht. Als Empfehlung für gezieltere Wahlkampagnen schlug Galasso vor, negative Wahlwerbung während Fußballspielen und positive während Spielfilmen zu zeigen. Zudem empfahl er, mehr Frauen in die Planung von Wahlkampagnen einzubeziehen.

\section{Fiskalische Institutionen: Ausgabepolitik und Zahlungsausfälle}

Am Beispiel des Bundeslandes Sachsen-Anhalt untersuchte Peter Haug die Wirkung vertikaler Geldzuweisungen auf die Effizienz kommunaler Ausgabepolitik. Die Annahme war, dass Wähler rational kalkulieren und davon ausgehen, dass eine Erhöhung der Finanzzuweisungen ihren Nutzen steigert und sie in geringerem Maße bereit sind, zur Finanzierung staatlicher Leistungen beizutragen. Entsprechend sei zu erwarten, dass die Ausgabepolitik in ärmeren Kommunen effizienter als in reicheren Kommunen sei. Untersucht wurden Ausgaben für Angestellte, Verkehr, Erholungsangebote, Kinderbetreuung sowie die Zahl der Kinder in Grundschulen und die Bevölkerungsentwicklung. Dabei zeige sich, dass die Ausgabepolitik reicher Kommunen weniger effizient als armer ist. In armen Kommunen würde zudem eine fiskalische Illusion, die systematische Überschätzung des Volumens der Geldzuweisungen durch die Bürger, die Effizienz weiter steigern.

Martin Weder untersuchte in einer 104 Fälle vergleichenden Länderstudie für den Zeitraum von 1980 bis 2009 den Einfluss von Regierungshandeln auf die Wahrscheinlichkeit eines Zahlungsausfalls von Staaten. Im untersuchten Zeitraum sei es einmal jährlich zu einem Zahlungsausfall gekommen, der jeweils mit dem Verlust des Zugangs zu den Kapitalmärkten sowie im Vorfeld mit einem negativen Wirtschaftswachstum verbunden sei. Die Untersuchung zeigte, dass ein hohes Wirtschaftswachstum und die politischen Institutionen den stärksten negativen Einfluss auf die Wahrscheinlichkeit des Eintritts eines Zahlungsausfalls haben. Während sich mithilfe der Ausgabenpolitik, die Wahrscheinlichkeit nicht reduzieren lasse, seien Maßnahmen auf der Einnahmeseite sehr wohl dazu geeignet. Aller- 
dings ermögliche es der Datensatz nicht, zu ergründen, ob es sich bei diesen Maßnahmen um Steuererhöhungen, eine Verbreiterung der Steuerbasis oder eine besser Durchsetzung der Steuerpflicht handele.

\section{,Institutions and Politicians Matter}

Bezüglich der ersten eingangs von Heinemann identifizierten, die Konferenzen tragenden Säule, die Analyse des Einflusses von Institutionen auf Politik, lässt sich resümieren:, institutions matter'. Alle Analysen der Wirkung expliziter und impliziter fiskalischer Regeln, des SWP, der Schuldenbremse, von, divided governments', Finanzräten und Regierungshandeln auf nachhaltige öffentliche Haushalte der jeweils untersuchten Einheiten, diagnostizieren prinzipiell eine Verschuldung bremsende Wirkung von Institutionen. Dabei hängt die jeweilige Wirkung von weiteren Faktoren ab, insbesondere dem jeweiligen Design der Instrumente.

Auch für die zweite Säule der Konferenz, die Analyse der Bedeutung von Politikern als Personen für Politik, lässt sich feststellen: ,politicians matter'. Eine Reihe, aber nicht alle, der vorgestellten Papiere wies Korrelationen zwischen biografischen Merkmalen von Politikern und spezifischen Politiken nach. Auch für die parteipolitische Zugehörigkeit von Politikern und ihrem Regierungshandeln wurden für die Haushalts- und Wirtschaftspolitik Korrelationen nachgewiesen. Aber auch die Papiere, die sich mit den Auswahlverfahren politischer Entscheidungsträger beschäftigten, zeigen, dass in diesem seltener bestellten Forschungsfeld noch erheblicher Forschungsbedarf besteht.

\section{Neue Reihe}

\section{Genshagener Schriften - Europa politisch denken}

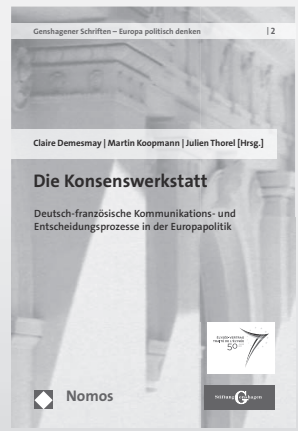

Bestellen Sie jetzt telefonisch unter 07221/2104-37 Portofreie Buch-Bestellungen unter www.nomos-shop.de/21029 denken, Bd. 2)

\section{Die Konsenswerkstatt}

Deutsch-französische Kommunikations- und Entscheidungsprozesse in der Europapolitik Herausgegeben von Claire Demesmay, Martin Koopmann und Julien Thorel

2013, 231 S., brosch., 36,- -€ ISBN 978-3-8487-0528-3

(Genshagener Schriften - Europa politisch

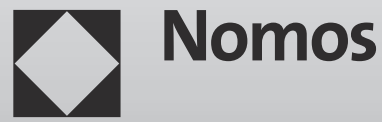

